Résumés des conférences et travaux

\title{
Histoire et philologie de l'Inde méridionale
}

\section{Appasamy Murugaiyan}

\section{OpenEdition \\ Journals}

\section{Édition électronique}

URL : https://journals.openedition.org/ashp/319

DOI : 10.4000/ashp.319

ISSN : 1969-6310

Éditeur

Publications de l'École Pratique des Hautes Études

\section{Édition imprimée}

Date de publication : 1 octobre 2008

Pagination : 319-321

ISSN : 0766-0677

\section{Référence électronique}

Appasamy Murugaiyan, "Histoire et philologie de l'Inde méridionale », Annuaire de l'École pratique des hautes études (EPHE), Section des sciences historiques et philologiques [En ligne], 139 | 2008, mis en ligne le 26 novembre 2008, consulté le 12 juillet 2021. URL : http://journals.openedition.org/ashp/319 ; DOl : https://doi.org/10.4000/ashp.319 


\title{
HISTOIRE ET PHILOLOGIE DE L'INDE MÉRIDIONALE
}

\author{
Chargé de conférences : M. Appasamy MurugaiYan
}

Programme de l'année: I. Initiation à la lecture des textes épigraphiques tamouls. Histoire de la langue tamoule. - II. Initiation au comparatisme des langues dravidiennes du Sud (suite).

\section{Initiation à la lecture des textes épigraphiques tamouls. Histoire de la langue tamoule}

Cette année nous avons poursuivi nos travaux sur l'émergence d'un langage épigraphique tamoul et avons approfondi des questions de sémantique lexicale. L'étude de la sémantique lexicale nous paraît d'une importance capitale dans le cadre de nos travaux engagés depuis plusieurs années à savoir : la désambiguïsation et la compréhension des textes épigraphiques et la constitution d'une base de données épigraphiques. Nous avons examiné deux axes : 1) l'extension sémantique des unités lexicales et 2) l'étude du style manipravāla et de son importance sociolinguistique. Pour ce faire, nous avons présenté l'analyse d'un corpus épigraphique très varié constitué des inscriptions de stèles funéraires et des temples couvrant une période $\mathrm{du} \mathrm{VI}^{\mathrm{e}}$ au XII ${ }^{\mathrm{e}}$ siècles.

L'extension sémantique des lexèmes conduit d'un sens inhérent à une interprétation afférente qui résulte d'une contrainte contextuelle. Certains lexèmes, en plus du sens normalement attesté, acquièrent de nouvelles significations qui couvrent des champs sémantiques spécialisés. Dans certains cas les deux valeurs sont employées, d'où souvent une ambiguitté d'interprétation. Nous les illustrerons avec quelques exemples.

Le lexème nu $\bar{a}$ l, s'emploie avec les sens de « homme, époux, serviteur, guerrier, brave, virile, messager mâle, bravoure » (DEDR.399). Parallèlement nous notons $\bar{a} l$ qui signifie, « régner, gouverner, contrôler, administrer » (DEDR.5157). Dans la construction suivante kongrai nățtu pācārạal pakaimatar, le terme $\bar{a} l$ qui relève de deux catégories syntaxiques nom et verbe, peut être interprété de deux façons, soit « pakaimatar le héros de pācāru du district de koṇrai », soit « pālāciriyar qui règne sur pācāru du district de koṇrai » (Chhs.1971/91).

Nous rencontrons deux termes de parentés qui couvrent un champ sémantique étendu. Il s'agit de makkal et marumakkal : 1) makkal, le pluriel de *maka > makan, signifie « enfant, jeune d'un animal, fils, fille, jeune âge » et aussi « chef, homme, guerrier » (DEDR.4616). 2) marumakkal, le pluriel de marumakan, désigne « neveu, descendant, membre d'un clan » (DEDR.4715). Ces deux termes s'emploient dans ces textes épigraphiques avec un sens spécialisé contextuellement : 1) renvoie à un membre de l'entourage immédiat du roi ou à ses descendants 2) désigne en général « un chef du clan, chef du village ou un serviteur », et aussi, « un héros ou des guerriers ». Par ailleurs, les termes makan et makkal sont également employés avec le sens principal de « fils de » (Dharmapuri.1972/3; Chhs.1971/86). L'emploi simultané des deux valeurs rend l'interprétation ambiguë. 
Nous rencontrons des noms propres comme poñarampañār, poñmāntan̄ār. Ces patronymes sont préfixés de pon et ce dernier signifie «or, fer, métaux, joyaux, richesse » (DEDR.4570). Ce préfixe est employé dans ces noms comme un titre et pourrait signifier « propriétaire de bétail ». Ces noms ne sont attestés que dans les textes épigraphiques de la région de Cheñkam. Ce lexème pon de l'or, dans son évolution sémantique a intégré « le bétail, le troupeau de vaches » dans son champ sémantique, qui représente la richesse au sein d'une société de bergers et d'éleveurs. Cette spécialisation sémantique bien que disparue en tamoul, est encore attestée dans au moins deux langues dravidiennes à tradition orale : en kota comme « bœuf» (nom propre) et en toda, signifie « bracelet en or dans la laiterie » (toda : gold bangle in dairy) [DEDR.4570]. Le sens des unités lexicales examinées ici, se définit comme un ensemble d'acceptions regroupées autour d'un sens central «prototypique » selon la théorie de la sémantique du prototype.

Nous avons ensuite attiré l'attention sur l'importance du style maniprvāḷa dans les textes épigraphiques. Comme nous avons déjà eu l'occasion de le mentionner lors de nos travaux antérieurs, le maniprvāla des textes épigraphiques n'est pas reconnu comme un phénomène sociolinguistique essentiel dans l'histoire de la langue tamoule. La plus ancienne inscription en écriture tamoule (vatteleluttu ancienne) découverte à ce jour, qui date du $\mathrm{v}^{\mathrm{e}}$ siècle (inscription de Pūlāṅkuricci) montre que la diffusion et l'intégration des éléments lexicaux prakrits et sanskrits sont, dès cette époque, très avancés, comme le démontre l'exemple suivant : attikōyattār « officier en charge du régiment des éléphants »(Sircar, 1966, p.127; āvanam.1. 1991). Ce terme est composé de hastikośa « officier en charge du régiment des éléphants », le cas oblique tamoul -tt-; et le suffixe tamoul de pluriel et honorifique - $\bar{a} r$-. Il faut noter dans cet exemple que l'adaptation du terme hastikośa > attikoyam, correspond à la règle phonologique tamoule : suppression de l'aspirée /h/ en position initiale; assimilation de la sifflante /s/ en dentale / $\mathrm{t} /$; l'ajout de la consonne /-m/ terminaison des noms neutres en tamoul et enfin alternance entre $/ \mathrm{s} / \mathrm{et} / \mathrm{y} /$ par le phénomène de l'affaiblissement de la sifflante /ś/ en semi-voyelle palatale /y/.

Au cours du temps, le nombre des emprunts, les champs sémantiques qu'ils couvrent et leur importance culturelle ne cessent d'augmenter. Nous verrons ci-dessous deux épithètes des rois cholas. Chaque titre est composé d'éléments indo-aryens et non pas de leurs calques en tamoul.

pānțiya kulāsañi < pānțiya + kula + aśani qui veut dire littéralement « Pantya + dynastie royale + (coup de) foudre », d'où le sens de « destructeur de la dynastie pandya ». L'expression suivante en est une variante : pānțiya kulāntaka < pānțiya + kula + antaka qui se traduit mot à mot par « Pantya + dynastie royale + tueur de, qui supprime », avec le sens de " celui qui met fin à la dynastie de Pandyas ».

Le contact linguistique est principalement appréhendé par l'observation des emprunts lexicaux, par le degré de leur intégration et par leur fréquence. Ces exemples témoignent d'un contact très ancien et intense entre le tamoul, le sanskrit, et le prakrit - la durée du contact ayant été suffisamment longue pour permettre une telle diffusion phonologique et morphologique. Il est généralement reconnu que tout élément d'une langue peut être emprunté. Néanmoins, sur le plan du lexique, il semble que certaines catégories soient plus propices à l'emprunt et que certains domaines sémantiques, tels 
que les concepts culturels ou techniques, se diffusent plus rapidement. Par ailleurs, les éléments qui sont sémantiquement adaptables en tamoul, sont souvent calqués. L'emploi de l'indo-aryen s'est maintenu et s'est accru pendant de nombreux siècles, témoignant probablement de l'existence d'un bilinguisme avec le tamoul. Bien que l'impact de ce contact soit diversement interprété, on ne peut pas éluder la question de l'influence de l'indo-aryen comme une des langues de communication administrative ainsi que sur le vocabulaire épigraphique tamoul.

\section{Initiation au comparatisme des langues dravidiennes du Sud}

La syntaxe comparée des langues dravidiennes, encore trop peu explorée, gagnerait à être précisée par des études diachroniques. Au regard de la quantité de données dont on dispose en dravidien pour la phonologie et la morphologie, notre objectif est d'apporter quelques éléments nouveaux aux travaux antérieurs en syntaxe historique. L'ordre des mots, un des domaines encore peu exploré en dravidien, occupe une place importante dans les travaux de changement linguistique et de typologie linguistique. Nous avons donc poursuivi les travaux sur l'ordre des constituants afin d'identifier, à travers plusieurs strates temporelles successives, l'évolution de l'ordre des mots en tamoul.

Les études menées sur un corpus des textes épigraphiques tamoul nous ont permis de souligner que l'aspect pragmatique y joue un rôle important. Toujours dans une perspective diachronique et compte tenu de la diversité des structures énonciatives, nous avons étudié la place des objets de don. Cette analyse, qui s'appuie sur le principe de structure informationnelle et l'ordre des constituants, laisse apparaître deux structures distinctes. D'une part, il existe des compléments d'objet direct qui se trouvent à gauche du prédicat verbal, occupant la place habituellement réservé à l'objet syntaxique, marqué ou non au cas accusatif. D'autre part, nous avons relevé, avec une fréquence élevée dans le corpus épigraphique tamoul, des objets de don apparaissant à droite du prédicat verbal. Ce syntagme nominal disloqué à droite, généralement hors syntaxe, n'est pas régi par le verbe, mais il lui est lié sur le plan pragmatique. Nous appellerons ces objets disloqués des objets externes. Ce type de dislocation à droite est accompagné du phénomène du flottement de quantificateur, qui lui aussi se trouve à droite au lieu de précéder le déterminé, créant ainsi une symétrie structurale :

mūvēntavēlān [.....]kuțtta [...] vilakku oṇru ... : nom propre.datif - donner.participe relatif-lampe-une « Muvenda Velan a offert une lampe ». Ce qui laisse supposer que la dislocation de l'objet de don à droite n'est pas une anomalie structurale, mais bien le résultat d'une stratégie énonciative. Il faut noter aussi que tous les objets de dons ne sont pas obligatoirement des compléments d'objets directs. Ces variations dans l'ordre des mots ne peuvent se comprendre qu'à la lumière de phénomènes corrélés, comme le changement historique (contact de langues, vestiges de structures anciennes, etc.) ou l'interaction entre phénomènes syntaxiques, sémantiques et énonciatifs. 